\title{
Matriz de colágeno xenógena para tratamento de recessões gengivas: revisão de
}

\section{literatura}

\author{
Xenogenous collagen matriz to treatment of gingival recessions: literature review \\ Matriz de colágeno xenógeno para el tratamiento de las recesiones gingivales: revisión de la
}

literatura

Recebido: 26/01/2021 | Revisado: 31/01/2021 | Aceito: 02/02/2021 | Publicado: 09/02/2021

\author{
Nicolly Cardoso Fernandes \\ ORCID: https://orcid.org/0000-0002-5543-1073 \\ Universidade Estadual de Londrina, Brasil \\ E-mail: nicolly09.nc@gmail.com \\ Natan Santos Massambani \\ ORCID: https://orcid.org/0000-0001-9536-2984 \\ Universidade Estadual de Londrina, Brasil \\ E-mail: natanmassambani@hotmail.com \\ Luciana Prado Maia \\ ORCID: https://orcid.org/0000-0001-5697-2587 \\ Universidade Estadual de Londrina, Brasil \\ E-mail: lucianapmaia@gmail.com \\ Fernanda Akemi Nakanishi Ito \\ ORCID: https://orcid.org/0000-0002-7849-5846 \\ Universidade Estadual de Londrina, Brasil \\ E-mail: fenakanishi@uel.br \\ Maria Beatriz Bergonse Pereira Pedriali \\ ORCID: https://orcid.org/0000-0001-8595-7108 \\ Universidade Estadual de Londrina, Brasil \\ E-mail: mbeatrizpedriali@uel.br \\ Priscila Paganini Costa \\ ORCID: https://orcid.org/0000-0002-0250-5905 \\ Universidade Estadual de Londrina, Brasil \\ E-mail: pripaganini@uel.br
}

\begin{abstract}
Resumo
A utilização da matriz de colágeno xenógena (MCX) tem sido investigada como uma alternativa para o recobrimento radicular no tratamento da recessão gengival, principalmente em áreas que necessitam de aumento de tecido queratinizado e a fim de diminuir a morbidade do paciente e o tempo cirúrgico. O objetivo desta revisão de literatura é analisar a utilização da matriz de colágeno xenógena como uma forma de tratamento para recobrimento das recessões gengivais. Foi realizada uma busca bibliográfica dos ensaios clínicos controlados e aleatórios, publicados entre 2010 a 2020, que avaliaram o tratamento de recessão gengival unitária ou múltipla utilizando a matriz de colágeno xenógena, sendo selecionados 14 ensaios clínicos de acordo com os critérios de inclusão. Os resultados apontaram que a combinação da MCX associada ao reposicionamento coronário (RC) é uma alternativa viável no tratamento da recessão gengival, apresentando resultados semelhantes quando comparado ao enxerto de tecido conjuntivo subepitelial (ETCS+RC). A utilização da MCX associada ao RC tem se mostrado eficiente quanto ao ganho de tecido queratinizado e aumento de espessura gengival no tratamento de recessões gengivais Tipo I (RT1), e pode ser uma alternativa ao ETCS em pacientes com limitada área doadora palatal e também reduzir a morbidade do paciente e o tempo operatório.
\end{abstract}

Palavras-chave: Recessão gengival; Cirurgia Plástica; Periodontia.

\begin{abstract}
The use of the xenogenous collagen matrix (MCX) was investigated as an alternative for root coverage in the treatment of gingival recession, especially in areas that require an increase in keratinized tissue and in order to decrease the patient's morbidity and surgical time. The purpose of this literature review is to analyze the use of the xenogen collagen matrix as a form of treatment for covering gingival recessions. A bibliographic search of controlled and randomized clinical trials was carried out, before 2010 to 2020, which evaluated the treatment of single or multiple gingival recession using a xenogen collagen matrix, and 14 clinical trials were selected according to the inclusion criteria. The results showed that the combination of MCX associated with coronary repositioning (CR) is a viable alternative in the treatment of gingival recession, similar results when compared to the subepithelial connective
\end{abstract}


tissue graft (ETCS + RC). The use of MCX associated with CR has been effective in gaining keratinized tissue and increasing gingival thickness in the treatment of Type I (RT1) gingival recessions, and can be an alternative to ETCS in patients with limited palatal donor area and also reduce patient morbidity and operative time.

Keywords: Gingival recession; Plastic surgery; Periodontics.

\section{Resumen}

Se investigó el uso de la matriz de colágeno xenógeno (MCX) como alternativa de cobertura radicular en el tratamiento de la recesión gingival, especialmente en áreas que requieren un aumento de tejido queratinizado y con el fin de disminuir la morbilidad y el tiempo quirúrgico del paciente. El propósito de esta revisión de la literatura es analizar el uso de la matriz de colágeno xenógeno como forma de tratamiento para cubrir las recesiones gingivales. Se realizó una búsqueda bibliográfica de ensayos clínicos controlados y aleatorizados, antes de 2010 a 2020, que evaluaron el tratamiento de la recesión gingival única o múltiple utilizando una matriz de colágeno xenógeno, y se seleccionaron 14 ensayos clínicos según los criterios de inclusión. Los resultados mostraron que la combinación de MCX asociada al reposicionamiento coronario (CR) es una alternativa viable en el tratamiento de la recesión gingival, resultados similares al compararlo con el injerto de tejido conectivo subepitelial (ETCS + RC). El uso de MCX asociado con $\mathrm{RC}$ ha sido eficaz para ganar tejido queratinizado y aumentar el grosor gingival en el tratamiento de recesiones gingivales de Tipo I (RT1), y puede ser una alternativa al ETCS en pacientes con área donante palatina limitada y también reducir morbilidad del paciente y tiempo operatorio.

Palabras clave: Recesión gingival; Cirugía plástica; Periodoncia.

\section{Introdução}

A recessão gengival é caracterizada pela alteração da margem gengival no sentido apical (Pini-Prato et al., 2010). Pode ser desencadeada por diversos fatores associados ou não, sendo os fatores predisponentes (mal posicionamento dentário, fenótipo periodontal fino, estreita faixa de gengiva inserida, inserção de freios e bridas, fenestração e deiscência óssea), precipitantes (trauma oclusal, margem e restauração/ coroas protéticas, escovação traumática), e também os fatores fisiológicos (movimentação ortodôntica) (Saade \& Bassani, 2017).

A recessão frequentemente tem como consequência a alteração estética, exposição do cemento radicular, desenvolvimento de lesão cervical não cariosa e hipersensibilidade dentinária (Pini-Prato et al., 2010). Quando as recessões gengivais apresentam comprometimento da saúde bucal e qualidade de vida do paciente um tratamento/ intervenção é indicado (Chambrone \& Tatakis, 2015).

O diagnóstico da recessão gengival é realizado através de uma classificação, que irá definir o plano de tratamento e prognóstico adequado para o defeito. A nova classificação das doenças e condições do periodonto (Caton et al., 2018) propõe uma nova forma para classificar as recessões gengivais (Cairo et al., 2011). Nesta classificação, Recessão tipo 1 (RT1) é descrita como a recessão sem perda de inserção interproximal; RT2 é a recessão associada com a perda de inserção clínica interproximal, onde a quantidade da perda seja menor ou igual à perda de inserção clínica na face vestibular; RT3 é descrita como a recessão associada com a perda de inserção clínica interproximal, onde a quantidade de perda é maior que a perda de inserção clínica na face vestibular (Cairo et al., 2011).

Grandes avanços têm sido apresentados nas técnicas cirúrgicas periodontais com o objetivo de obter a cobertura radicular completa e diminuição da hipersensibilidade, melhorando a estética e devolvendo a função. As recessões podem ser tratadas utilizando diversas técnicas cirúrgicas como o enxerto gengival livre (EGL), retalhos periodontais reposicionados sozinhos ou combinados usando ou não aloenxertos ou membranas (Cairo et al., 2008).

O enxerto de tecido conjuntivo subepitelial (ETCS) é considerado padrão ouro no recobrimento radicular. Atualmente também tem sido utilizado com sucesso no manejo de tecidos moles em regiões ao redor de implantes (Silva et al., 2020). Entretanto, está associado a fatores importantes como a morbidade do paciente e realização da cirurgia em duas áreas (Nunn \& Miyamoto, 2013). Além disso, pode estar limitado quanto ao tamanho, forma e espessura (Barakat \& Dayoub, 2020).

Nesse sentido, os pesquisadores buscam por materiais alternativos que proporcionam a mesma ou semelhante qualidade, como o uso de substitutos de tecidos moles (STM). Um desses biomateriais é a matriz tridimensional colágena 
xenógena (MCX), que é uma matriz de colágeno de origem suína (Geistlich Biomaterials GmbH, Baden-Baden, Alemanha), desenvolvida para a regeneração de tecidos moles e utilizada especificamente para a substituição dos transplantes autólogos (McGuire \& Scheyer, 2010). É indicada principalmente quando há contraindicação para obtenção do ETCS, devido ao fenótipo gengival fino, tamanho e espessura do enxerto ou anatomia do palato, e em casos em que o objetivo do operador seja aumentar a faixa de gengiva queratinizada e reduzir a morbidade (Cardaropoli et al., 2012).

Uma das principais vantagens em se utilizar a MCX é a ausência de dois sítios cirúrgicos e também por não possuir limite no tamanho do enxerto, fazendo com que ocorra a redução do tempo operatório e da medicação pós-operatória, em relação ao ETCS. Porém, como desvantagem a chance de se obter um resultado inferior quanto ao recobrimento radicular, comparado ao ETCS, é maior (Tonetti et al., 2018).

Considerando a utilização de biomateriais, a MCX pode ser um substituto do enxerto de tecido conjuntivo autógeno, a fim de diminuir o desconforto do paciente e reduzir o tempo cirúrgico sem perder a qualidade dos resultados clínicos. Vale ressaltar sua indicação, principalmente em áreas extensas, já que não possui tamanho limitado comparado ao enxerto gengival (Schimitt et al., 2015).

Diante disso, este trabalho tem como objetivo realizar uma revisão de literatura sobre a utilização da matriz de colágeno xenógena como uma forma de tratamento das recessões gengivais.

\section{Metodologia}

Trata-se de um estudo qualitativo de revisão de literatura (Pereira et al., 2018). Foi realizada uma busca bibliográfica no PubMed utilizando os termos: "mucograft and gingival recession" e "xenogenic collagen matrix and gingival recession". Foram incluídos ensaios clínicos controlados e aleatórios, publicados no entre 2010 e 2020, em Inglês, que avaliaram o tratamento de recessão gengival unitária ou múltipla utilizando a matriz de colágeno xenógena. Foram excluídos os estudos que utilizaram a matriz como tratamento em regiões de implantes dentários. Foram encontrados 31 artigos e 14 ensaios clínicos foram selecionados de acordo com os critérios de inclusão e exclusão.

\section{Revisão de Literatura}

As recessões gengivais frequentemente estão associadas com a insatisfação estética e com a hipersensibilidade dentinária resultante da exposição da superfície radicular ao ambiente oral (Wennstrom, 1996). O seu desenvolvimento geralmente pode ser associado com a dificuldade de controlar o biofilme dental e/ ou trauma durante a escovação (Rejapakse et al., 2007), podendo implicar diretamente na função, estética e conforto do paciente (Merijohn, 2016). Rios et al. (2020), em sua estimativa, verificaram uma maior incidência e progressão da recessão gengival em pacientes adultos.

O tratamento das recessões gengivais, muitas vezes, é realizado por meio de técnicas de cirurgia periodontal plástica, como retalho posicionado lateralmente (Grupe, 1966), retalho posicionado coronalmente (Bernimoulin et al., 1975), enxerto subepitelial de tecido conjuntivo (Langer \& Langer, 1985), retalho semilunar (Tarnow, 1986), técnica da dupla papila (Harris, 1992), regeneração tecidual guiada (RTG) (Pini-Prato et al., 1992), técnica do túnel (Zabalegui et al., 1999), e técnicas cirúrgicas que sofreram modificações (Zadeh, 2011).

Contudo, para suprir a necessidade de um segundo sítio e diminuir a comorbidade, técnicas cirúrgicas alternativas vêm sendo propostas e comparadas a fim de obter a redução da recessão gengival, ganho de inserção clínica e tecido queratinizado, além de compatibilidade de cor e textura com um resultado estético satisfatório (Cardaropoli et al., 2012). Diante disso, a MCX foi desenvolvida com as propriedades qualitativas e de segurança e podem ser utilizadas na substituição do tecido autólogo no recobrimento radicular (Thoma et al., 2009).

A MCX, conhecida comercialmente como Mucograft ${ }^{\circledR}$ (Geistlich Biomaterials GmbH, Baden-Baden, Alemanha), é 
uma matriz de origem suína, bilaminar, composta de colágeno tipo I e III puro (Herford et al., 2010). Esta matriz é obtida por meio de processos controlados e padronizados, sem manipulação química ou ligações cruzadas, e cuidadosamente purificada para evitar antigenicidade (Ghanaati et al., 2011).

O enxerto subepitelial de tecido conjuntivo é considerado o padrão ouro no tratamento de recessões gengivais (Zadeh, 2011). Assim sendo, a literatura científica apresenta uma série de estudos clínicos randomizados e controlados que se propuseram a comparar a MCX ao ETCS.

\subsection{Utilização da matriz de colágeno xenógena comparada a técnica de retalho reposicionado coronalmente.}

No estudo comparativo de Jepsen et al. (2013), foi realizada a associação da MCX+RC no tratamento de recessões gengivais localizadas comparado apenas ao RC. Ao todo, foram 45 indivíduos e 90 recessões Classe I e II de Miller (1985), RT1 de Cairo (2011), randomizadas. A avaliação dos resultados mostrou que o grupo da MCX+RC apresentou uma média de $75,29 \%$ de cobertura radicular, enquanto o grupo RC sozinho mostrou média de $72,66 \%$ de cobertura radicular no acompanhamento de 6 meses ( $\mathrm{p}=0,169)$ Quanto ao aumento na largura média de tecido queratinizado, o resultado também foi melhor no grupo teste $(1,97$ a 2,90 mm) em relação ao grupo controle $(2,00$ a 2,57 mm), além do ganho em espessura gengival, que foi estatisticamente maior $(\mathrm{p}=0,036)$ no grupo teste $(\mathrm{MCX}+\mathrm{RC})$.

Em 2016, Moreira et al. avaliaram o tratamento para recessão gengival unitária classe I e II de Miller (1985), RT1 de Cairo (2011), a partir da comparação do RC sozinho ou combinado com a MCX. A amostra contou com 40 participantes e a média de recobrimento radicular foi de 77,2\% no grupo teste (RC+MCX) e de 72,1\% no grupo controle (RC) em 6 meses de pós operatório. O grupo teste também apresentou um melhor ganho de tecido queratinizado, mesmo não sendo estatisticamente significante. E quanto à análise estética do procedimento realizada pelos pacientes, ambos os grupos apresentaram melhoras significativas.

Da mesma forma, o estudo de Jepsen et al. (2017) também relatou os resultados obtidos após 6 meses e 3 anos para o tratamento de cobertura radicular em 18 pacientes com recessões gengivais localizadas do tipo I e II de Miller (1985), RT1 de Cairo (2011), contabilizando 36 defeitos de recessão. No acompanhamento de 6 meses, observou-se uma média significante (p $=0,0039)$ no recobrimento radicular no grupo teste RC+MCX de 89,9\% e 91,76\% em 3 anos. No grupo controle (RC), a porcentagem de recobrimento radicular foi de $83,7 \%$ e $82,8 \%$ em 6 meses e 3 anos respectivamente.

Recentemente, Rotundo et al. (2019) compararam a associação da MCX+RC (grupo teste) ao RC (grupo controle) no tratamento de recessões gengivais múltiplas $\geq 2 \mathrm{~mm}$. O estudo incluiu 24 indivíduos, e 61 recessões foram randomizadas e acompanhadas. Na avaliação de 1 ano, os resultados mostraram que a redução da profundidade da recessão gengival foi de 2,3 $\pm 0,7$ para $0,3 \pm 0,4 \mathrm{~mm}$ no grupo teste e de $2,6 \pm 1,0$ para $0,6 \pm 0,3 \mathrm{~mm}$ no grupo controle. Em relação à espessura gengival, os resultados analisados mostraram um aumento significativo no grupo teste em comparação ao grupo controle, nos quais se verificou que $47 \%$ dos sítios tratados somente com o $\mathrm{RC}$ mostraram uma diminuição da espessura < $1 \mathrm{~mm}$ no acompanhamento de 1 ano de pós operatório, em comparação com 21\% dos sítios tratados com a MCX.

Os resumos dos estudos mencionados estão na Tabela 1. 
Tabela 1: Utilização da Matriz de Colágeno Xenógena comparada a Técnica de Retalho Reposicionado Coronalmente.

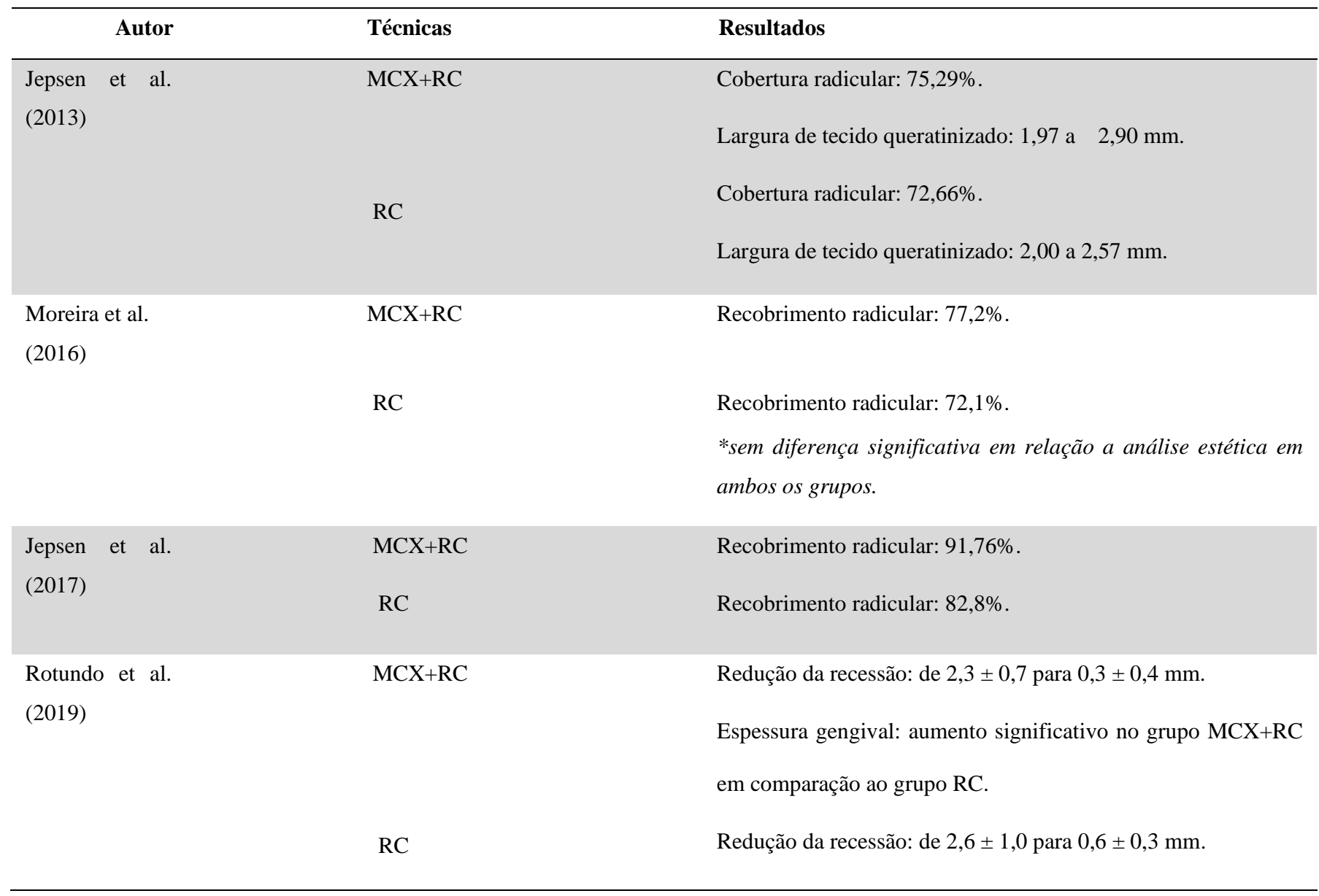

Fonte: Autores.

\subsection{Utilização da matriz de colágeno xenógena comparada ao enxerto de tecido conjuntivo subepitelial associado com a} técnica de retalho reposicionado coronalmente.

McGuire e Scheyer (2010) avaliaram o uso da matriz de colágeno xenógena associado ao reposicionamento coronário (MCX+RC) (grupo teste) em comparação com o enxerto de tecido conjuntivo subepitelial também associado ao reposicionamento coronário (ETCS+RC) (grupo controle). Este estudo contou com 25 indivíduos (17 mulheres e 8 homens) e as avaliações foram realizadas aos 6 meses e 1 ano. Foram avaliados os seguintes parâmetros: largura do tecido queratinizado, profundidade de sondagem e tamanho da recessão. Na avaliação de 6 meses, a profundidade da recessão foi, em média, 0,52 mm para o grupo teste e 0,10 mm para o grupo controle, sem diferença estatística. Já a profundidade de sondagem apresentou uma alteração significativa entre os grupos teste e o controle, com uma média de 2,62 mm de ganho no grupo teste e 3,10 mm de ganho no grupo controle, e com uma diferença de $(\mathrm{p}=0,0062)$. Na avaliação de 1 ano, o grupo MCX+RC apresentou cobertura radicular estatisticamente menor que o grupo ETCS+RC $(88,5 \%$ e 99,3\%, respectivamente) $(\mathrm{p}=0,0313)$, enquanto que a largura de tecido queratinizado foi similar (1,34 mm e 1,26 mm para os grupos teste e controle, respectivamente). Não houve diferenças significativas nas avaliações relatadas pelos indivíduos em relação à estética e à dor.

Após 5 anos de acompanhamento deste mesmo estudo (McGuire \& Scheyer, 2016), apenas 17 pacientes participaram da avaliação. A porcentagem média de recobrimento radicular para o grupo teste foi de 77,6\% e 95,5\% para o grupo controle $(\mathrm{p}=0,1563)$. A largura de tecido conjuntivo apresentou uma média de mais de $3 \mathrm{~mm}$ e a coloração do tecido permaneceu semelhante nos dois grupos.

Cardaropoli et al. (2012) também compararam a matriz de colágeno xenógena associada ao reposicionamento 
coronário $(\mathrm{MCX}+\mathrm{RC})$ e o enxerto de tecido conjuntivo subepitelial associado reposicionamento coronário (ETCS+RC) no tratamento de recessões gengivais. Esse estudo contou com a participação de 18 indivíduos ( 9 homens e 9 mulheres). Vinte e duas recessões gengivais unitárias classes I e II de Miller (1985), RT1 de Cairo (2011), foram randomizadas nos grupos teste e controle. O acompanhamento foi de 12 meses e o percentual de cobertura radicular no grupo MCX+RC e no grupo ETCS+RC foi de $94,32 \%$ e $96,97 \%$, respectivamente. Foi observado também, ganho de espessura de 1,23 mm no grupo teste e de 1,27 $\mathrm{mm}$ no grupo controle, sem diferença estatística.

Da mesma forma, Chevalier et al. (2017) investigaram o uso da MCX comparada ao ETCS no tratamento da recessão gengival. A amostra contou com 4 pacientes adultos que continham no total 17 recessões unitárias e múltiplas classe I de Miller (1985) e RT1 de Cairo (2011). A média de redução da recessão nos grupos ETCS e MCX foi de 2 mm, não havendo nenhuma diferença entre os dois grupos. Os pacientes puderam julgar em uma escala visual a dor durante o pós-operatório, e a pontuação média para o ETCS foi de 3,4 enquanto que para a MCX foi de 2,4, mostrando ser uma opção para diminuir o risco de comorbidade do paciente.

O desafio por meio do manejo clínico no tratamento de recessões múltiplas está interligado com as limitações de tamanho, formato e espessura do enxerto de tecido conjuntivo autólogo. Pensando nisso, Tonetti et al. (2018) realizaram um estudo para testar a não inferioridade da MCX quando comparada com o ETCS combinados com o reposicionamento coronário (RC) para o recobrimento radicular em recessões múltiplas. O estudo contou com 187 indivíduos e 485 recessões gengivais randomizadas e acompanhadas pelo período de 6 meses. $\mathrm{O}$ resultado obtido neste período em relação à cobertura radicular foi de 1,7 $\pm 1,1 \mathrm{~mm}$ para MCX e 2,1 $\pm 1,0 \mathrm{~mm}$ para, apresentando uma superioridade significante. Porém, no quesito espessura gengival, a MCX mostrou ser superior com o ganho de $0,5 \mathrm{~mm}$, enquanto no ETCS houve a perda de $0,1 \mathrm{~mm}$. Além disso, a avaliação realizada pelos pacientes mostrou que a percepção de dor foi menor durante os 7 dias de pós-operatório no grupo da MCX. O estudo concluiu que a matriz de colágeno é considerada uma alternativa eficiente na cobertura radicular de múltiplas recessões, regeneração periodontal, aumento de tecido queratinizado, evitando dois sítios cirúrgicos, menor tempo cirúrgico e menor tempo de recuperação quando comparado ao enxerto autólogo.

Recentemente Barakat et al. (2020) realizaram um estudo, no qual comparou o uso do ETCS+RC com a MCX+RC no tratamento das recessões gengivais classe I e II de Miller (1985), RT1 de Cairo (2011). Vinte pacientes participaram do estudo, contabilizando 40 recessões gengivais. Os resultados utilizando a MCX associada ao ganho em espessura foram superiores no grupo teste, sendo de $1,35 \mathrm{~mm}$, enquanto no grupo controle a espessura obtida foi de 1,30 mm em 12 meses de acompanhamento.

Suzuki et al. (2020) também realizaram um estudo com o intuito de investigar o uso da MCX combinada com reposicionamento coronário estendido (RCE), comparada ao ETCS para o recobrimento radicular em recessão gengival bilateral RT1 de Cairo (2011). A amostra contou com 18 indivíduos e 36 recessões foram randomizadas e acompanhadas. Os resultados avaliados durante 3 e 6 meses não demonstraram diferenças significativas em ambos os grupos quanto ao recobrimento radicular. Um questionário foi aplicado aos pacientes, no qual os mesmos realizaram uma avaliação sobre a estética. E, em ambos os grupos, também não foram observadas diferenças estatísticas. O estudo a partir de seus resultados mostra que a MCX, quando comparada ao ETCS para o recobrimento radicular, apresenta resultados semelhantes.

Os resumos dos estudos mencionados estão na Tabela 2. 
Tabela 2: Utilização da Matriz de colágeno Xenógena comparada ao Enxerto de Tecido Conjuntivo Subepitelial com a Ténica de Retalho Reposicionado Coronalmente.

\begin{tabular}{|c|c|c|}
\hline Autor & \multirow{2}{*}{$\begin{array}{l}\text { Técnicas } \\
\mathrm{MCX}+\mathrm{RC}\end{array}$} & \multirow{2}{*}{$\begin{array}{l}\text { Resultados } \\
\text { Cobertura radicular: em média de } 88,5 \% \text {. } \\
\text { Largura de tecido queratinizado: } 1,34 \mathrm{~mm} \text {. }\end{array}$} \\
\hline $\begin{array}{l}\text { McGuire, Scheyer } \\
\text { (2010) }\end{array}$ & & \\
\hline & $\mathrm{ETCS}+\mathrm{RC}$ & $\begin{array}{l}\text { Cobertura radicular: em média de } 99,3 \% \text {. } \\
\text { Largura de tecido queratinizado: } 1,26 \mathrm{~mm} \text {. }\end{array}$ \\
\hline $\begin{array}{l}\text { McGuire, Scheyer } \\
\text { (2016) }\end{array}$ & $\mathrm{MCX}+\mathrm{RC}$ & Recobrimento radicular: $77,6 \%$ \\
\hline & $\mathrm{ETCS}+\mathrm{RC}$ & $\begin{array}{l}\text { Recobrimento radicular: } 95,5 \% \text {. } \\
\text { Largura de tecido queratinizado: uma média de mais de } 3 \mathrm{~mm} \text { nos } \\
\text { grupos MCX e ETCS. }\end{array}$ \\
\hline $\begin{array}{l}\text { Cardaropoli et al. } \\
\text { (2012) }\end{array}$ & $\mathrm{MCX}+\mathrm{RC}$ & $\begin{array}{l}\text { Recobrimento radicular: } 94,32 \% \text {. } \\
\text { Aumento de espessura: } 1,23 \mathrm{~mm} \text {. }\end{array}$ \\
\hline & $\mathrm{ETCS}+\mathrm{RC}$ & $\begin{array}{l}\text { Recobrimento radicular: } 96,97 \% \text {. } \\
\text { Aumento de espessura: } 1,27 \mathrm{~mm} \text {. } \\
\text { *sem diferença estatística no aumento de espessura. }\end{array}$ \\
\hline Chevalier et al. (2017) & $\mathrm{MCX}+\mathrm{RC}$ & $\begin{array}{l}\text { Redução da recessão: Em amobos os grupos grupo (ETCS e } \\
\text { MCX) foi de } 2,00 \mathrm{~mm} \text {. }\end{array}$ \\
\hline & $\mathrm{ETCS}+\mathrm{RC}$ & *sem diferença na redução da recessão. \\
\hline Tonetti et al. (2018) & $\mathrm{MCX}+\mathrm{RC}$ & $\begin{array}{l}\text { Cobertura radicular: } 1,7 \pm 1,1 \mathrm{~mm} \text { para } \mathrm{MCX} \text { e } 2,1 \pm 1,0 \mathrm{~mm} \text { para } \\
\text { ETCS. }\end{array}$ \\
\hline & $\mathrm{ETCS}+\mathrm{RC}$ & $\begin{array}{l}\text { Espessura gengival: MCX ganhou de } 0,5 \mathrm{~mm} \text {, enquanto no ETCS } \\
\text { houve a perda de } 0,1 \mathrm{~mm} .\end{array}$ \\
\hline Barakat et al. (2020) & $\begin{array}{l}\mathrm{MCX}+\mathrm{RC} \\
\mathrm{ETCS}+\mathrm{RC}\end{array}$ & $\begin{array}{l}\text { Ganho de espessura gengival: } 1,35 \mathrm{~mm} . \\
\text { Ganho de espessura gengival: } 1,30 \mathrm{~mm} \text {. }\end{array}$ \\
\hline Sukuki et al. (2020) & $\begin{array}{l}\mathrm{MCX}+\mathrm{RC} \\
\mathrm{ETCS}+\mathrm{RC}\end{array}$ & $\begin{array}{l}\text { No recobrimento radicular e também na avaliação estética não } \\
\text { houve diferenças significativas em ambos os grupos. }\end{array}$ \\
\hline
\end{tabular}

Fonte: Autores.

\subsection{Utilização da matriz de colágeno xenógena comparada a outras técnicas cirúrgicas.}

Outras técnicas também foram avaliadas junto da MCX. No estudo realizado por Cieślik-Wegemund et al. (2016), a técnica de túnel utilizada no recobrimento radicular foi comparada na combinação da técnica do túnel + ETCS (grupo controle) e o túnel + MCX (grupo teste), em recessões múltiplas classe I e II de Miller (1985), RT1 de Cairo (2011). A amostra contou com 28 pacientes e 106 recessões foram randomizadas e controladas durante 6 meses. A cobertura radicular média neste 
período foi de $95 \%$ no grupo controle e $91 \%$ no grupo teste. A média de espessura gengival aumentou nos dois grupos.

Em um estudo subsequente, Cieślik-Wegemund et al. (2018) avaliaram e compararam a eficácia clínica do uso da técnica do túnel com a MCX no recobrimento de recessões múltiplas na maxila ou mandíbula. A população deste estudo foi formada por 14 indivíduos. O resultado mostrou-se promissor com a porcentagem média de cobertura radicular de 96,8\% na maxila e 81,3\% na mandíbula pelo período de 6 meses de acompanhamento.

Neste outro ensaio clínico randomizado, Sangiorgio et al. (2017) investigaram o uso da MCX comparando com proteína derivada da matriz de esmalte (PDME) no tratamento de recessões unitárias classe I e II de Miller (1985), RT1 de Cairo (2011). A amostra contou com 68 participantes, que foram divididos em quatro grupos: RC; RC+MCX; RC+PDME; e RC+MCX+PDME. A análise dos resultados mostrou que a cobertura radicular completa no grupo RC+PDME foi em média de 70,59\%, estatisticamente superior aos grupos de RC+MCX (52,94\%), RC (23,53\%), e RC+MCX+PDME (51,47\%). Porém, houve um ganho significativo ( $\mathrm{p}<0,05)$ em espessura gengival de $2,58 \mathrm{~mm}$ a 2,93 $\mathrm{mm}$ nos grupos que foram tratados com a MCX. Na avaliação estética, não houve diferença estatisticamente significativa entre os grupos.

Os resumos dos estudos mencionados estão na Tabela 3.

Tabela 3: Utilização da Matriz de Colágeno Xenógena comparada a Outras Ténicas Cirúrgicas.

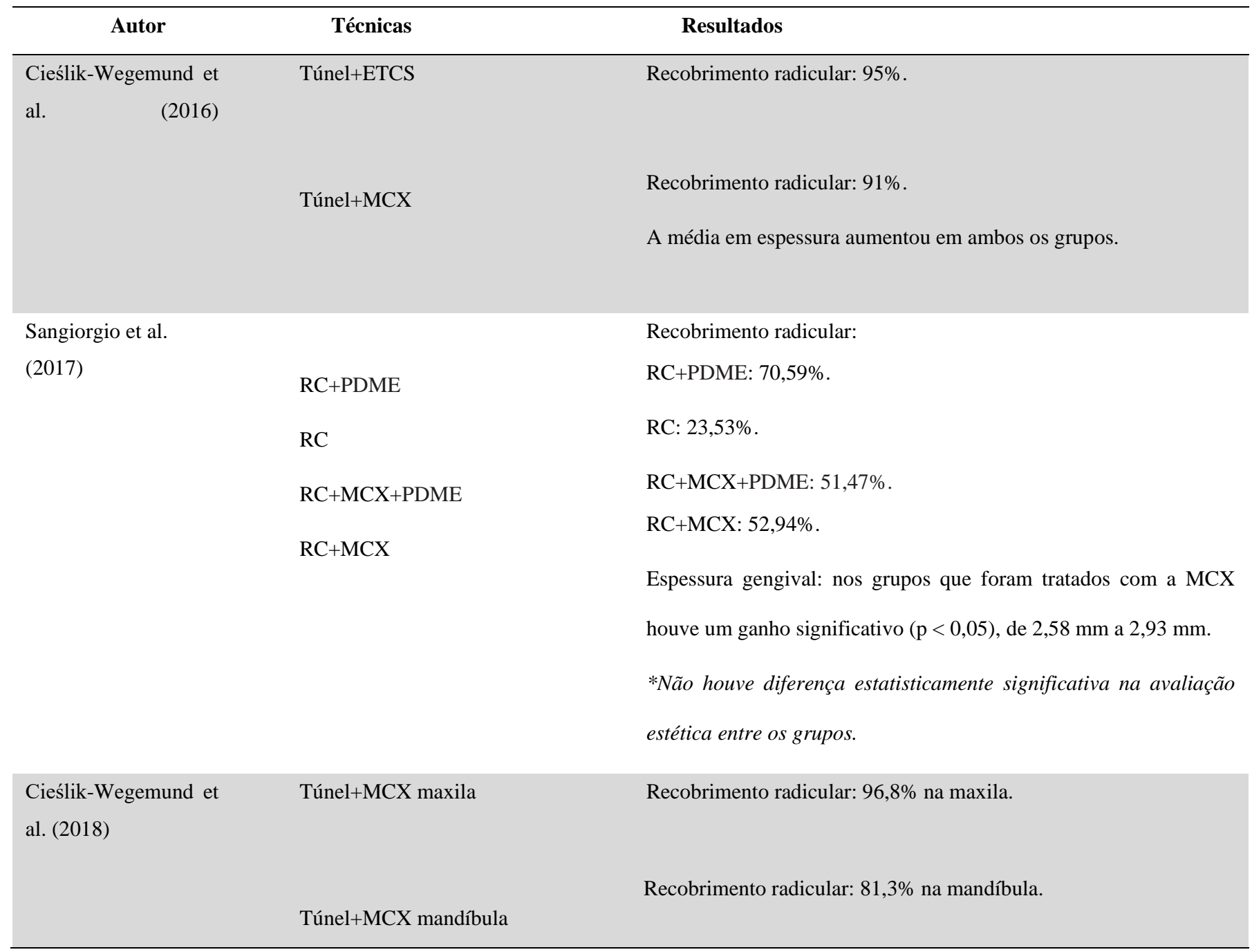




\section{Discussão}

Existem diversas técnicas cirúrgicas capazes de aumentar o tecido queratinizado e recobrir uma recessão gengival. A técnica do EGL é uma delas, na qual enxertos epitelizados são removidos geralmente do palato (Pini-Prato et al., 2018). Podese utilizar também a técnica do ETCS, considerada o padrão ouro, mas assim como na técnica anterior, a remoção do enxerto do palato causa uma maior morbidade ao paciente, além do tempo cirúrgico relativamente longo (Cieślik-Wegemund et al., 2016).

Devido a isso, o substituto de tecido mole vem sendo cada vez mais utilizado com o intuito de reduzir o desconforto pós-operatório do paciente, diminuir o tempo cirúrgico e segurança durante o procedimento. Dessa forma, a comunidade científica tem conduzido estudos a fim de observar a eficácia e a segurança desses substitutos moles (Schimitt et al., 2013).

A MCX, comercialmente denominada de Mucograft $®$, é um dispositivo obtido por meio de uma fabricação controlada e padronizada com certificação veterinária para evitar reações antigênicas, que visa o tratamento de recessões gengivais (Nevins et al., 2011). A MCX possui bicamada de células funcionais, sendo constituída por uma fina camada de células, e a outra sendo mais porosa representando $90 \%$ do volume da matriz (Sanz et al., 2009). As células presentes na matriz atuam como recrutadoras de receptores e células, fazendo com que o processo da cicatrização seja acelerado (Ghanaati et al., 2011).

Uma das formas para se realizar o tratamento da recessão gengival é por meio da técnica do RC, com resultados clínicos satisfatórios (Sangiogio et al., 2017). Porém, a associação da MCX a essa técnica tem apresentado benefício adicional no recobrimento radicular. Vários estudos se propuseram a avaliar a eficácia clínica desta associação no tratamento de recessões unitárias e múltiplas classe I e II de Miller (1985), RT1 de Cairo (2011), como os estudos de Jepsen et al. (2013), Moreira et al. (2016), Jepsen et al. (2017) e Rotundo et al. (2019).

Moreira et al. (2016) observaram que a adição da MCX à técnica do RC está associada a uma maior taxa na cobertura radicular quando comparada ao RC sozinho. Corroboram com estes achados os estudos de Jepsen et al. (2013) e o estudo de Jepsen et al. (2017). Já o estudo de Rotundo et al. (2019) apresentou resultados semelhantes quanto à redução da recessão gengival, mas houve um ganho de espessura maior no grupo da $\mathrm{MCX}+\mathrm{RC}$ assim como de Jepsen et al. (2013), que também apresentaram maior espessura no grupo teste.

Na comparação do ETCS com a MCX ambos associados ao RC, McGuire e Scheyer (2010) realizaram o primeiro ensaio clínico. Além de ser estudada e comparada por meio dos parâmetros clínicos como, ganho de tecido queratinizado, nível de inserção clínica, redução da profundidade de sondagem, a cor e a textura dos tecidos também estão sendo analisadas pelo paciente (Cardaropoli et al., 2012). O resultado na porcentagem de recobrimento radicular, altura da recessão gengival e diminuição na profundidade de sondagem no estudo de McGuire e Scheyer (2010) foram melhores no grupo controle quando comparado ao grupo teste após 1 ano. Já o ganho em largura foi expressivo neste tempo de acompanhamento, mostrando resultados próximos em ambos os grupos. Corroboraram com estes achados, os estudos de McGuire e Scheyer (2016), CieślikWegemund et al. (2016) e Tonetti et al. (2018). Somente em relação à espessura gengival, Tonetti et al. (2018) mostraram melhores resultados no grupo da MCX+RC bem como Barakat et al. (2020). Por outro lado, os estudos de Cardaropoli et al. (2012), Chevalier et al. (2017) e Suzuki et al. (2020) apresentaram resultados semelhantes, sem diferença estatística, na porcentagem de recobrimento radicular.

A literatura relata que o ETCS associado com o RC é considerado o padrão ouro no tratamento de recessões gengivais tanto unitárias quanto múltiplas devido as suas vantagens como cobertura radicular completa, aumento em espessura e maior estabilidade. Porém, uma segunda área operatória, aumenta o tempo de cirurgia e torna o pós-operatório mais doloroso ao paciente (Tonetti et al., 2018). Na avaliação realizada pelos pacientes em relação à satisfação estética e aos níveis da dor, foram observados resultados semelhantes em ambas às técnicas utilizando ETCS e a MCX (McGuire \& Scheyer, 2010; 
Barakat \& Dayoub, 2020; \& Sukuki et al. 2020).

Porém, nos estudos de Chevalier et al. (2017) e de Tonetti et al. (2018) houve divergências quanto à percepção de dor do paciente em relação ao procedimento utilizando ETCS. Ambos relataram que a maior percepção de dor está associada à técnica de ETCS, principalmente onde as suturas foram realizadas no palato.

A literatura diz que a MCX pode sofrer um encolhimento aproximadamente de 30\% a 65\% posterior aos 6 meses de pós operatório (Schimitti et al., 2013). Porém, após 5 anos de tratamento, McGuire e Scheyer (2016) fizeram uma nova avaliação e relataram que os sítios tratados com enxerto de tecido conjuntivo subepitelial permaneceram inalterados, ou seja, 100\% de recobrimento. Já nos sítios com a MCX, a cobertura radicular aumentou de $66 \%$ para 100\%, além de ter mantido a largura de tecido queratinizado com uma média acima de $3 \mathrm{~mm}$. Dessa forma, mostrou-se, por meio dos parâmetros, que ao longo do tempo a diferença entre as terapias não apresentava mudança significativa.

Para investigar se a adição da PDME à MXC apresentava benefício adicional ao recobrimento radicular, Sangiorgio et al. (2017) compararam quatro grupos no tratamento de recessões unitárias classe I e II de Miller (1985), RT1 de Cairo (2011): RC, RC+MCX, RC+MDE, RC+MCX+PDME. Neste estudo, os autores observaram, diante das variadas associações, que as técnicas que obtiveram uma maior cobertura radicular foram realizadas com o RC combinadas com a PDME e MCX em grupos distintos, pois a PDME não mostrou benefício no uso associado com a MCX para o recobrimento radicular. O ganho em espessura foi expressivo nos grupos em que foram tratados com a MCX, comparado aos demais grupos.

\section{Considerações Finais}

É importante salientar que o enxerto de tecido conjuntivo ainda é o tratamento padrão ouro para o recobrimento radicular. Porém, diante dos estudos e resultados apresentados, a utilização da matriz de colágeno xenógena quando associada ao retalho reposicionado coronário, representa uma alternativa viável tanto na redução da recessão gengival, como no ganho de tecido queratinizado e aumento de espessura no tratamento de recessão gengival, obtendo resultados clínicos e estéticos próximo ao retalho reposicionado associado com o enxerto de tecido conjuntivo, além de reduzir o tempo cirúrgico e a morbidade do paciente. Outros estudos são necessários para verificar a estabilidade da matriz de colágeno xenógena no recobrimento radicular a longo prazo.

\section{Referências}

Barakat, H., \& Dayoub, S. (2020). Treatment of miller type I and II gingival recession defects using three-dimensional porcine collagen matrix with coronally advanced flap: A randomized clinical split-mouth trial (a 1-year follow-up). Indian J. Dent Res, 31, 209-216.

Bernimoulin, J. P., Luscher, B., \& Muhlermann, H. R. (1975). Coronally repositioned periodontal flap. Clinical evaluation after one year. J. of Clinical Periodontology, 2(1), 1-13.

Cairo, F., Nieri, M., Cincinelli, S., Mervelt, J., \& Pagliaro, U. (2011). The interproximal clinical attachment level to classify gingival recessions and predict root coverage outcomes: an explorative and reliability study. J. of Clinical Periodontology, 38(7), 661-666.

Cairo, F., Pagliaro, U., \& Nieri, M. (2008). Treatment of gingival recession with advanced coronary flap procedures: a systematic review. J. Clin Periodontol, $35,136-62$.

Cardaropoli, D., Tamagnone, L., Rofredo, A., \& Gaveglio, L. (2012). Treatment of gingival recession defects using coronally advanced flap with a porcine collagen matrix compared to coronally advanced flap with connective tissue graft: A randomized controlled clinical trial. J. Periodontol, 83, 321-8.

Caton, J. G., Armitage, G., Berglundh, T., Chapple, I. L. C., Jepsen, S., Kornman, K. S., Mealey, B. L., Papapanou, P. N., Sanz, M., \& Tonetti, M. S. (2018). A new classification scheme for periodontal and peri-implant diseases and conditions - Introduction and key changes from the 1999 classification. $J$. of Periodontology, 45, 1-8.

Chambrone, L., \& Tatakis, D. N. (2015). Periodontal soft tissue root coverage procedures: a systematic review from the AAP Regeneration Workshop. J. of Periodontology, 86(2), 8-51.

Chevalier, G., Cherkaoui, S., Kruk, H., Bensaid, X., \& Danan, M. (2017). Xenogeneic collagen matrix versus connective tissue graft: Case series of various gingival recession treatments. Int J Periodontics Dent Restorative, 37, 117-23. 
Cieslik-Wegemound, M., Wieruka-Mlynarczyk, B., Tanasiewincz, M., \& Gilowski, L. (2016). Tunnel technique with collagen matrix compared with connective tissue graft for treatment of periodontal recession: A randomized clinical trial. J. Periodontol, 87, 436-43.

Cieslik-Wegemound, M., Candotto, V., \& Wierucka-Młynarczyk, B. (2018). Coverage of multiple recessions using the tunnel technique and a collagen matrix in the maxilla or mandible: a 6-month study. J. Biol Regul Homeost Agents, 32, 1-10.

Ghanaati, S., Schlee, M., Webber, M. J., Willershausen, I., Barbeck, M., Balic, E., Görlach, C., Stupp, S. I., Sader, R. A., \& Kirkpatrick, C. J. (2011). Evaluation of the tissue reaction to a new bilayered collagen matrix in vivo and its translation to the clinic. Biomedical materials (Bristol, England), 6(1), 015010.

Grupe, H. E. (1966). Modified technique for the sliding flap operation. J. of periodontology, 37(6), 491-495.

Harris, R. J. (1992). The connective tissue and partial thickness double pedicle graft: a predictable method of obtaining root coverage. J. of periodontology, 63(5), 477-486

Herford, A. S., Akin, L., Cicciu, M., Maiorana, C., \& Boyne, P. J. (2010). Use of a porcine collagen matrix as an alternative to autogenous tissue for grafting oral soft tissue defects. Journal of oral and maxillofacial surgery: official journal of the American Association of Oral and Maxillofacial Surgeons, 68(7), $1463-1470$.

Jepsen, K., Jepsen, S., Zucchelli, G., Stefanini, M., de Sanctis, M., Baldini, N., Greven, B., Heinz, B., Wennström, J., Cassel, B., Vignoletti, F., \& Sanz, M. (2013). Treatment of gingival recession defects with a coronally advanced flap and a xenogeneic collagen matrix: a multicenter randomized clinical trial. Journal of clinical periodontology, 40(1), 82-89.

Jepsen, K., Stefanini, M., Sanz, M., Zucchelli, G., \& Jepsen, S. (2017). Long-Term Stability of Root Coverage by Coronally Advanced Flap Procedures. Journal of periodontology, 88(7), 626-633.

Langer, B., \& Langer, L. (1985). Subepithelial connective tissue graft technique for root coverage. J. of Periodontology, 56(2), 715-720.

McGuire, M. K., \& Scheyer, E. T. (2010). Xenogeneic collagen matrix with coronally advanced flap compared to connective tissue with coronally advanced flap for the treatment of dehiscence-type recession defects. J. Periodontol, 81(8), 1108-17.

McGuire, M. K., \& Scheyer, E. T. (2016). Long-Term Results Comparing Xenogeneic Collagen Matrix and Autogenous Connective Tissue Grafts With Coronally Advanced Flaps for Treatment of Dehiscence-Type Recession Defects. J. Periodontol, 87(3), 221-7.

Merijohn, G. K. (2016). Management and prevention of gingival recession. Periodontology 2000, 71(1), 228-242.

Miller, P. D. (1985). A classification of marginal tissue recession. International Journal of Periodontics and Restorative Dentistry, 5(2), 8-13.

Moreira, A., Santamaria, M. P., Silvério, K. G., Casati, M. Z., Nociti Junior, F. H., Sculean, A., \& Sallum, E. A. (2016). Coronally advanced flap with or without porcine collagen matrix for root coverage: a randomized clinical trial. Clinical oral investigations, 20(9), 2539-2549.

Nevins, M., Nevins, M. L., Kim, S. W., Schupbach, P., \& Kim, D. M. (2011). The use of mucograft collagen matrix to augment the zone of keratinized tissue around teeth: a pilot study. The International journal of periodontics \& restorative dentistry, 31(4), 367-373.

Nunn, M. E., \& Miyamoto, T. (2013). Advanced coronary flaps (CAF) plus connective tissue graft (CTG) are the gold standard for the treatment of Miller class I and II gingival defects. J. Evid Based Dent Pract, 13, 157-9.

Pereira, A. S., Shitsuka, D. M., Parreira, F. J., \&Shitsuka, R. (2018). Metodologia do trabalho científico. UAB/NTE/UFSM. https://repositorio.ufsm.br/bitstream/handle/1/15824/Lic_Computacao_Metodologia-Pesquisa-Cientifica. pdf.

Pini-Prato, G., Franceschi, D., Cairo, F., Nieri, M., \& Rotundo, R. (2010). Classification of dental surface defects in areas of gingival recession. Journal of periodontology, 81(6), 885-890.

Pini-Prato, G., Magnani, G., \& Chambrone, L. (2018). Long-term evaluation (20 years) of the outcomes of coronally advanced flap in the treatment of single recession-type defects. J. Periodontology, 89, 265-274.

Pini-Prato, G., Tinti, C., Vincenzi, G., Magnani, C., Cortellini, P., \& Clauser, C. (1992). Guided tissue regeneration versus mucogingival surgery in the treatment 77 of human buccal gingival recession. J. of periodontology, 63(11), 919-928.

Rajapakse, P. S., McCracken, G. I., Gwynnett, E., Steen, N. D., Guentschu, A., \& Heasman, P. A. (2007). Does tooth brushing influence the development and progression of non-inflammatory gingival recession? A systematic review. J. Clin Periodontol, 34, 1046-1061.

Rios, F. S., Costa, R. S. A., Wagner, T. P., Christofoli, B. R., Goergen, J., Izquierdo, C., Jardim, J. J., Maltz, M. \& Haas, A. N. (2021). Incidence and progression of gingival recession over 4 years: A population-based longitudinal study. J Clin Periodontol, 48, 115-126.

Rotundo, R., Genzano, L., Patel, D., D'Aiuto, F., \& Nieri, M. (2019). Adjunctive benefit of a xenogenic collagen matrix associated with coronally advanced flap for the treatment of multiple gingival recessions: A superiority, assessor-blind, randomized clinical trial. Journal of clinical periodontology, 46(10), 10131023 .

Saade, J., \& Bassani, M. (2017). Cirurgia plástica periodontal- recobrimento radicular. <http://www.clinicasaade.com.br/wp-content/uploads/2013/05/14CONGR_vol5.pdf>

Sangiorgio, J., Neves, F., Rocha Dos Santos, M., França-Grohmann, I. L., Casarin, R., Casati, M. Z., Santamaria, M. P., \& Sallum, E. A. (2017). Xenogenous Collagen Matrix and/or Enamel Matrix Derivative for Treatment of Localized Gingival Recessions: A Randomized Clinical Trial. Part I: Clinical Outcomes. Journal of periodontology, 88(12), 1309-1318. 
Research, Society and Development, v. 10, n. 2, e17910212363, 2021

(CC BY 4.0) | ISSN 2525-3409 | DOI: http://dx.doi.org/10.33448/rsd-v10i2.12363

Sanz, M., Lorenzo, R., Aranda, J. J., Martin, C., \& Orsini, M. (2009). Clinical evaluation of a new collagen matrix (Mucograft prototype) to enhance the width of keratinized tissue in patients with fixed prosthetic restorations: a randomized prospective clinical trial. Journal of clinical periodontology, 36(10), 868-876.

Schimitt, C. M., Moest, T., Lutz, R., Wehrhan, F., Neukan, F. W., \& Schlegel, K. A. (2015). Long-term outcomes after vestibuloplasty with a porcine collagen matrix (Mucograft) versus the free gingival graft: a comparative prospective clinical trial. Clin. Oral Impl. Res, 1-9.

Schimitt, C. M., Tudor, C., Kiener, K., Wehrhan, F., Schimitt, J., Eitner, S., Agaimy, A., \& Schlegel, K. A. (2013). Vestibuloplasty: Porcine Collagen Matrix Versus Free Gingival Graft: A Clinical and Histologic Study. J. of periodontology, 84(7), 914-923.

Silva, R. A. R., Neto, L. F. A., Marcelino, K. P., Cardoso, L. C. L., Dantas, E. M., Barbosa, G. A. S., Macêdo, F. C., Gondim, A. L. M. F. (2020). Osteotomia segmentar da maxila em área estética para viabilizar reabilitação com implantes dentários: relato de caso. Research, Society and Development, 9(9).

Suzuki, K. T., de Jesus Hernandez Martinez, C., Suemi, M. I., Palioto, D. B., Messora, M. R., de Souza, S., Novaes, A. B., Jr, Chaves Furlaneto, F. A., \& Taba, M., Jr (2020). Root coverage using coronally advanced flap with porcine-derived acellular dermal matrix or subepithelial connective tissue graft: a randomized controlled clinical trial. Clinical oral investigations, 24(11), 4077-4087.

Tarnow, D. P. (1986). Semilunar coronally repositioned flap. J. of Clinical Periodontology, 13(3), 182-185.

Thoma, D. S., Benic, G. I., Zwahlen, M., Hammerle, C. H. F., \& Jung, R. E. (2009). A systematic review assessing soft tissue augmentation techniques. Clinical Oral Implants Research, 20, 146-165.

Tonetti, M. S., Cortellini, P., Pellegrini, G., Nieri, M., Bonaccini, D., Allegri, M., Bouchard, P., Cairo, F., Conforti, G., Fourmousis, I., Graziani, F., Guerrero, A., Halben, J., Malet, J., Rasperini, G., Topoll, H., Wachtel, H., Wallkamm, B., Zabalegui, I., \& Zuhr, O. (2018). Xenogenic collagen matrix or autologous connective tissue graft as adjunct to coronally advanced flaps for coverage of multiple adjacent gingival recession: Randomized trial assessing non-inferiority in root coverage and superiority in oral health-related quality of life. Journal of clinical periodontology, 45(1), 78-88.

Wennstrom, J. L. (1996). Mucogingival therapy. Ann Periodontol, 1, 671-701.

Zabalegui, I., Sicilia, A., Cambra J., Gil, J., \& Sanz, M. (1999). Treatment of multiple adjacent gingival recessions with the tunnel subepithelial connective tissue graft: a clinical report. The International journal of periodontics \& restorative dentistry, 19(2), 199-206.

Zadeh, H. H. (2011). Minimally invasive treatment of maxillary anterior gingival recession defects by vestibular incision subperiosteal tunnel access and platelet-derived growth factor BB. The International journal of periodontics \& restorative dentistry, 31(6), 653-660. 